\title{
Basic properties of a vortex in a noncentrosymmetric superconductor
}

\author{
N. Hayashi ${ }^{a}$, Y. Kato ${ }^{\text {b }}$, P. A. Frigeri ${ }^{a}$, K. Wakabayashi ${ }^{\text {a }}$, \\ M. Sigrist ${ }^{a}$ \\ ${ }^{a}$ Institut für Theoretische Physik, ETH-Hönggerberg, CH-8093 Zürich, Switzerland \\ ${ }^{\mathrm{b}}$ Department of Basic Science, University of Tokyo, Tokyo 153-8902, Japan
}

\begin{abstract}
We numerically study the vortex core structure in a noncentrosymmetric superconductor such as $\mathrm{CePt}_{3} \mathrm{Si}$ without mirror symmetry about the $x y$ plane. A single vortex along the $z$ axis and a mixed singlet-triplet Cooper pairing model are considered. The spatial profiles of the pair potential, local density of states, supercurrent density, and radially-textured magnetic moment density around the vortex are obtained in the clean limit on the basis of the quasiclassical theory of superconductivity.
\end{abstract}

Key words: $\mathrm{CePt}_{3} \mathrm{Si}$, Unconventional superconductivity, Vortex core, Local density of states, Core magnetization, Broken inversion symmetry

PACS: 74.20.Rp; 74.70.Tx; 74.25.Op

Much attention has been focused on the heavy fermion superconductor $\mathrm{CePt}_{3} \mathrm{Si}$, which has a noncentrosymmetric crystal structure without mirror symmetry about the $x y$ plane [1]. $\mathrm{CePt}_{3} \mathrm{Si}$ is an extreme type-II superconductor and the vortex structure of the mixed state in this system was recently studied by Kaur et al. [2] and Yip [3] on the basis of the Ginzburg-Landau theory and the London theory. In this paper, we investigate the vortex core structure on the basis of the quasiclassical theory of superconductivity [4], which enables us to calculate more microscopically the physical quantities such as the pair potential, local density of states, supercurrent density, and magnetic moment density. We consider a single vortex along the $z$ axis in the clean limit.

The noncentrosymmetricity (or the lack of inversion symmetry) leads to the mixture of Cooper pairing channels of different parity [5]. We consider the following superconducting order parameter in a singlet-triplet mixing form: $\hat{\Delta}_{k}=\left(\Psi \hat{\sigma}_{0}+\boldsymbol{d}_{k} \cdot \hat{\boldsymbol{\sigma}}\right) i \hat{\sigma}_{y}=\left[\Psi \hat{\sigma}_{0}+\Delta\left(-\tilde{k}_{y} \hat{\sigma}_{x}+\tilde{k}_{x} \hat{\sigma}_{y}\right)\right] i \hat{\sigma}_{y}$, with the $s$-wave pairing component $\Psi$ and the $\boldsymbol{d}$ vector $\boldsymbol{d}_{k}=\Delta\left(-\tilde{k}_{y}, \tilde{k}_{x}, 0\right)$. This $s+p$-wave 
pairing state is proposed for $\mathrm{CePt}_{3} \mathrm{Si}$ in Ref. [6]. Here, $\left(\hat{\sigma}_{x}, \hat{\sigma}_{y}, \hat{\sigma}_{z}\right)$ are the Pauli matrices in the spin space, $\hat{\sigma}_{0}$ is the unit matrix, and $\tilde{\boldsymbol{k}}=\left(\tilde{k}_{x}, \tilde{k}_{y}, \tilde{k}_{z}\right)=$ $(\cos \phi \sin \theta, \sin \phi \sin \theta, \cos \theta)$.

The lack of inversion symmetry here is incorporated through a Rashba-type spin-orbit coupling with a form proposed in Ref. [7]. It splits the Fermi surface into two ones (I and II) by lifting the spin degeneracy [6]. From the original Eilenberger equation for noncentrosymmetric superconductivity [8], we obtain two equations corresponding to these split Fermi surfaces I and II in the case of the above $s+p$-wave pairing state [9],

$$
i \boldsymbol{v}_{\mathrm{I}, \mathrm{II}} \cdot \boldsymbol{\nabla} \check{g}_{\mathrm{I}, \mathrm{II}}+\left[i \omega_{n} \check{\tau}_{3}-\check{\Delta}_{\mathrm{I}, \mathrm{II}}, \check{g}_{\mathrm{I}, \mathrm{II}}\right]=0,
$$

where $\check{\Delta}_{\mathrm{I}, \mathrm{II}}=\left[\left(\check{\tau}_{1}+i \check{\tau}_{2}\right) \Delta_{\mathrm{I}, \mathrm{II}}-\left(\check{\tau}_{1}-i \check{\tau}_{2}\right) \Delta_{\mathrm{I}, \mathrm{II}}^{*}\right] / 2, \Delta_{\mathrm{I}, \mathrm{II}}=\Psi \pm \Delta \sin \theta$ are the order parameters on the Fermi surfaces I and II, $\left(\check{\tau}_{1}, \check{\tau}_{2}, \check{\tau}_{3}\right)$ are the Pauli matrices in the particle-hole space, and the commutator $[\check{a}, \check{b}]=\check{a} \breve{b}-\check{b} \check{a}$. We neglect the vector potential in Eq. (1) assuming the extreme type-II superconductivity. We use units in which $\hbar=k_{\mathrm{B}}=1$.

The Green functions $\check{g}_{\mathrm{I}, \mathrm{II}}$ on the Fermi surfaces I and II are written as a matrix in the particle-hole space,

$$
\check{g}_{\mathrm{I}, \mathrm{II}}\left(\boldsymbol{r}, \tilde{\boldsymbol{k}}, i \omega_{n}\right)=-i \pi\left(\begin{array}{cc}
g_{\mathrm{I}, \mathrm{II}} & i f_{\mathrm{I}, \mathrm{II}} \\
-i \bar{f}_{\mathrm{I}, \mathrm{II}} & -g_{\mathrm{I}, \mathrm{II}}
\end{array}\right) \text {. }
$$

The regular Green function $\hat{g}$ as a matrix in the spin space is given by $[6,8,9]$

$$
\begin{aligned}
\hat{g} & =g_{\mathrm{I}} \hat{\sigma}_{\mathrm{I}}+g_{\mathrm{II}} \hat{\sigma}_{\mathrm{II}} \\
& =\frac{1}{2}\left(\begin{array}{cc}
g_{\mathrm{I}}+g_{\mathrm{II}} & -\bar{k}_{+}^{\prime}\left(g_{\mathrm{I}}-g_{\mathrm{II}}\right) \\
-\bar{k}_{-}^{\prime}\left(g_{\mathrm{I}}-g_{\mathrm{II}}\right) & g_{\mathrm{I}}+g_{\mathrm{II}}
\end{array}\right),
\end{aligned}
$$

with $\hat{\sigma}_{\mathrm{I}, \mathrm{II}}=\left(\hat{\sigma}_{0} \pm \overline{\boldsymbol{g}}_{k} \cdot \hat{\boldsymbol{\sigma}}\right) / 2$ and $\overline{\boldsymbol{g}}_{k}=\left(-\bar{k}_{y}, \bar{k}_{x}, 0\right)$. Here, $\bar{k}_{ \pm}^{\prime}=\bar{k}_{y} \pm i \bar{k}_{x}$ and $\overline{\boldsymbol{k}}=\left(\bar{k}_{x}, \bar{k}_{y}, 0\right)=(\cos \phi, \sin \phi, 0)$.

We consider a single vortex which has a form, $\Delta_{\mathrm{I}, \mathrm{II}}\left(r, \phi_{r} ; \theta\right)=[\Psi(r) \pm \Delta(r) \sin \theta] \exp \left(i \phi_{r}\right)$. Here, the real-space coordinates $\boldsymbol{r}=\left(r \cos \phi_{r}, r \sin \phi_{r}, 0\right)$, and the vortex center is situated at $\boldsymbol{r}=0$. The Fermi surface is assumed to be spherical, and the differences of the density of states and the Fermi velocity $\boldsymbol{v}_{\mathrm{I}, \mathrm{II}}$ between the two Fermi surfaces I and II are assumed to be small and are ignored. The results in this paper depend predominantly on the spin structure [Eq. (3)] and the gap structure on the 3D Fermi surfaces, and the Fermi-surface anisotropy would not lead to qualitatively different results as long as the spin and gap topologies are not altered. We numerically solve the gap equations given in Ref. $[6,8,9]$ and the Eilenberger equations in Eq. (1) self-consistently as in Ref. [10]. When 
solving the gap equations, we adopt the same values of parameters as used in Ref. [8]. Thus, both the pair potentials $\Delta$ and $\Psi$ are real and positive, and $|\Delta|>|\Psi|[8]$. From now on, $T_{\mathrm{c}}$ is the superconducting critical temperature and $\xi_{0}=v_{\mathrm{F}} / T_{\mathrm{c}}$ is the coherence length at zero temperature $\left(v_{\mathrm{F}}=\left|\boldsymbol{v}_{\mathrm{F}}\right|\right.$ is the Fermi velocity).
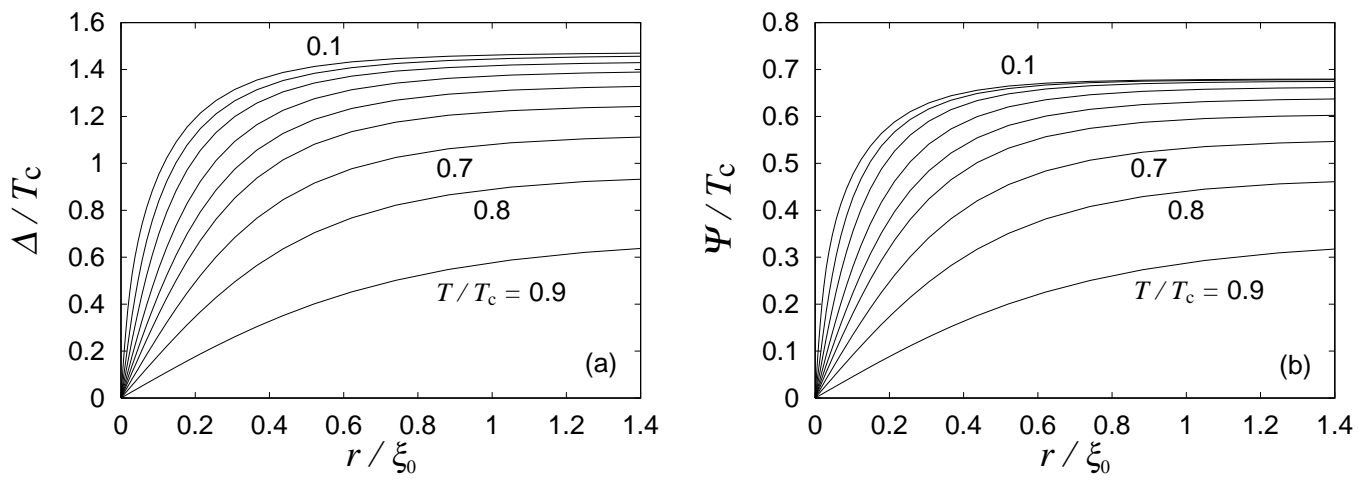

Fig. 1. Spatial profiles of the pair potentials. $T / T_{\mathrm{c}}=0.1-0.9$ from top to bottom by 0.1 step. (a) the $p$-wave component $\Delta$, and (b) the $s$-wave one $\Psi$.

In Fig. 1, we show the spatial profiles of the pair potentials $\Delta$ ( $p$-wave component) and $\Psi$ (s-wave one) around the vortex for several temperatures $T$. It is noticed that while the amplitude is different between $\Delta$ and $\Psi$, the characteristic recovery length (namely, the core radius) is the same for both.

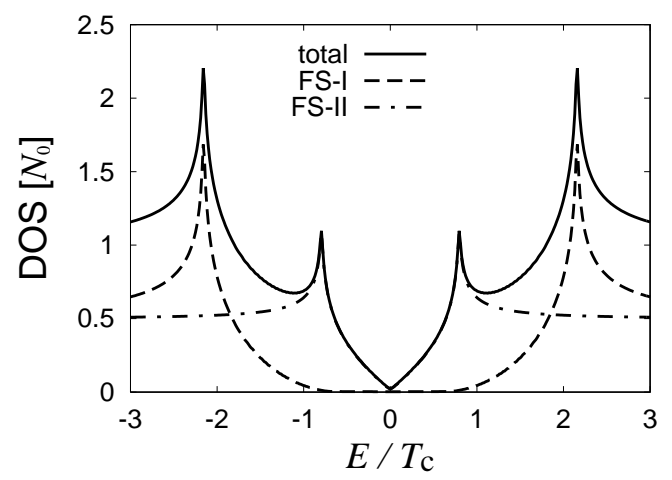

Fig. 2. The density of states in the bulk without vortices. $T / T_{\mathrm{c}}=0.1$ and $\eta=0.01 T_{\mathrm{c}}$.

The local density of states (per spin) is calculated by

$$
\begin{aligned}
N(E, \boldsymbol{r}) & =\frac{N_{0}}{2} \operatorname{Re}\left\langle\operatorname{Tr}\left[\hat{g}\left(i \omega_{n} \rightarrow E+i \eta\right)\right]\right\rangle \\
& =\frac{N_{0}}{2} \operatorname{Re}\left\langle g_{\mathrm{I}}+g_{\mathrm{II}}\right\rangle,
\end{aligned}
$$

where $\langle\cdots\rangle$ denotes the average over the Fermi surface, $N_{0}$ is the density of states per spin at the Fermi level, and $\eta(>0)$ is the energy smearing factor. 
Before going into the vortex bound states, let us see in Fig. 2 the density of states in the bulk without vortices. There are four gap edges (solid line). The system has two split Fermi surfaces I and II [8,9]. The two of the gap edges originate from the fully-gapped Fermi surface I (dashed line), and the other two originate from the line-node-gap Fermi surface II (dash-dotted line).

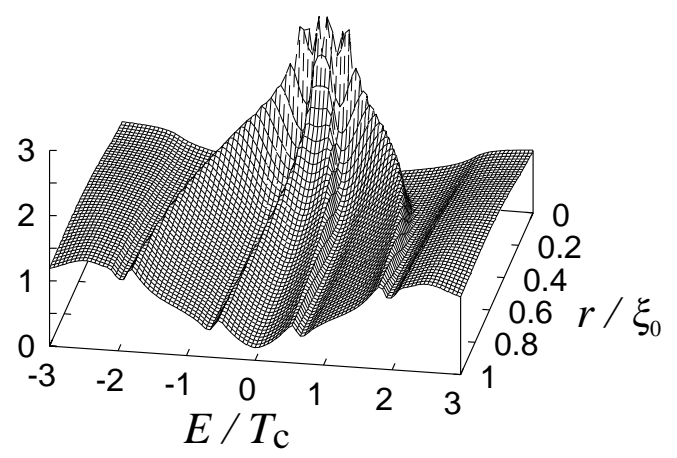

Fig. 3. The local density of states $N(E, r)$ inside the vortex core in units of $N_{0}$. $T / T_{\mathrm{c}}=0.1$ and $\eta=0.05 T_{\mathrm{c}}$. Large zero-bias peak at $(E, r)=(0,0)$ is truncated in the figure.

In Fig. 3, we show the local density of states inside the vortex core. There are four branches of peaks, which are related to the vortex bound states. The outer (inner) two branches originate from the vortex bound states of the quasiparticles on the Fermi surface I (II). Thus, the present spectra inside the vortex core in the clean limit possess the same structure as those in a two-gap superconductor.

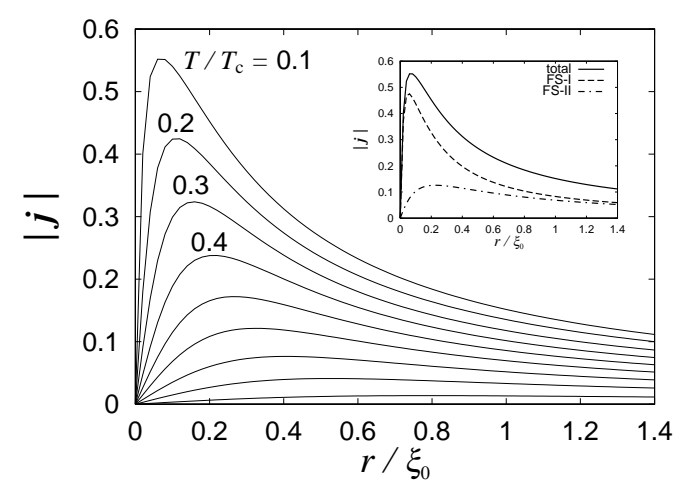

Fig. 4. Spatial profiles of the supercurrent density $|\boldsymbol{j}|$ in units of $2|e| v_{\mathrm{F}} N_{0} T_{\mathrm{c}}$. $T / T_{\mathrm{c}}=0.1-0.9$ from top to bottom by 0.1 step. Inset: $|\boldsymbol{j}|$ at $T / T_{\mathrm{c}}=0.1$ (solid line), the contribution of the Fermi surface I (dashed line), and that of the Fermi surface II (dash-dotted line).

In Fig. 4, we plot the supercurrent density $|\boldsymbol{j}|$, which is calculated by

$$
\boldsymbol{j}=e T \sum_{\omega_{n}} N_{0}\left\langle\boldsymbol{v}_{\mathrm{F}} \operatorname{Tr}\left[\hat{\sigma}_{0}(-i \pi \hat{g})\right]\right\rangle
$$




$$
=-i \pi e T \sum_{\omega_{n}} N_{0}\left\langle\boldsymbol{v}_{\mathrm{F}}\left(g_{\mathrm{I}}+g_{\mathrm{II}}\right)\right\rangle
$$

where $e$ is the electric charge of the quasiparticle. We have confirmed numerically that $|\boldsymbol{j}|$ decays as $\sim 1 / r$ far away from the core. $|\boldsymbol{j}|$ exhibits essentially the same structure as that in usual $s$-wave superconductors.

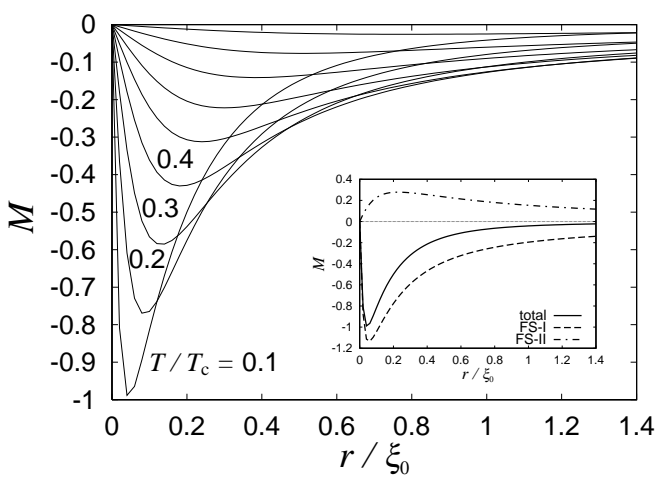

Fig. 5. The radial component of the magnetic moment density $\boldsymbol{M}$ around the vortex in units of $\mu_{\mathrm{B}} N_{0} T_{\mathrm{c}} . T / T_{\mathrm{c}}=0.1-0.9$ from bottom to top by 0.1 step. Inset: $\boldsymbol{M}$ at $T / T_{\mathrm{c}}=0.1$ (solid line), the contribution of the Fermi surface I (dashed line), and that of the Fermi surface II (dash-dotted line). The minus value means that the sense of $\boldsymbol{M}$ is toward the vortex center.

Finally, we investigate the magnetic moment density $\boldsymbol{M}$. The vortex-core magnetization in the present noncentrosymmetric system has been reported by Kaur et al. [2] and Yip [3]. Here, we calculate it to obtain numeric results at various temperatures by means of a more microscopic derivation. $\boldsymbol{M}$ is calculated by

$$
\boldsymbol{M}=\mu_{\mathrm{B}} T \sum_{\omega_{n}} N_{0}\langle\operatorname{Tr}[\hat{\boldsymbol{\sigma}}(-i \pi \hat{g})]\rangle
$$

where $\mu_{\mathrm{B}}$ is the magnetic moment of the quasiparticle. Substituting Eq. (3) into this, we obtain

$$
\begin{aligned}
& M_{x}=-i \pi \mu_{\mathrm{B}} T \sum_{\omega_{n}} N_{0}\left\langle\left(-\bar{k}_{y}\right)\left(g_{\mathrm{I}}-g_{\mathrm{II}}\right)\right\rangle, \\
& M_{y}=-i \pi \mu_{\mathrm{B}} T \sum_{\omega_{n}} N_{0}\left\langle\bar{k}_{x}\left(g_{\mathrm{I}}-g_{\mathrm{II}}\right)\right\rangle \\
& M_{z}=0 .
\end{aligned}
$$

We have confirmed numerically that the azimuthal component of $\boldsymbol{M}$ is zero around the vortex, namely $\boldsymbol{M}$ is aligned in the radial direction in the $x y$ plane. In Fig. 5, we show the spatial profiles of the radial component of $\boldsymbol{M}$. We checked that $|\boldsymbol{M}|$ decays as $\sim 1 / r$ far away from the core. The spatial profiles of $|\boldsymbol{M}|$ are similar to those of $|\boldsymbol{j}|$, but there is a difference because of 
their microscopic origin. While $|\boldsymbol{j}|$ is composed of $g_{\mathrm{I}}+g_{\mathrm{II}}$ in Eq. (5), $|\boldsymbol{M}|$ is composed of $g_{\mathrm{I}}-g_{\mathrm{II}}$ in Eqs. (7) and (8) [see also the insets of Figs. 4 and 5].

In conclusion, we calculated the vortex core structure in the noncentrosymmetric superconductor. We found that the singlet and triplet components of the order parameter have the same characteristic length (core radius) as each other. The local density of states exhibits the two-gap property in the clean limit, which is expected to be observed by STM $[11,12]$ in order to clarify the validity of the present mixed-parity pairing model. We mention here that impurity effects in the noncentrosymmetric system can be different from those in simple two-gap systems in general, therefore interesting phenomena in the density of states are expected when the system deviates from the clean limit. Detailed investigations on such impurity effects are left for future studies. The radially-textured magnetic moment appears around the vortex, which is expected to be observed directly by spin-polarized STM [13] in order to clarify the electronic structure [Eq. (3)] specific to the noncentrosymmetric superconductivity with the rotating spin on the Fermi surfaces as shown in a picture in Ref. [14].

We thank D. F. Agterberg for stimulating discussion. We are grateful for financial support from the Swiss Nationalfonds and the NCCR MaNEP. One

of us (N.H.) is also supported by 2003 JSPS Postdoctoral Fellowships for Research Abroad.

\section{References}

[1] E. Bauer, et al., Phys. Rev. Lett. 92 (2004) 027003.

[2] R. P. Kaur, D. F. Agterberg, M. Sigrist, Phys. Rev. Lett. 94 (2005) 137002.

[3] S. K. Yip, J. Low Temp. Phys. 140 (2005) 67.

[4] J. W. Serene, D. Rainer, Phys. Rep. 101 (1983) 221; and references therein.

[5] L. P. Gor'kov, E. I. Rashba, Phys. Rev. Lett. 87 (2001) 037004.

[6] P. A. Frigeri, D. F. Agterberg, I. Milat, M. Sigrist, cond-mat/0505108

[7] P. A. Frigeri, D. F. Agterberg, A. Koga, and M. Sigrist, Phys. Rev. Lett. 92 (2004) 097001.

[8] N. Hayashi, K. Wakabayashi, P. A. Frigeri, M. Sigrist, cond-mat/0504176.

[9] N. Hayashi, K. Wakabayashi, P. A. Frigeri, M. Sigrist, cond-mat/0510546.

[10] N. Hayashi, Y. Kato, M. Sigrist, J. Low Temp. Phys. 139 (2005) 79.

[11] H. F. Hess, R. B. Robinson, J. V. Waszczak, Phys. Rev. Lett. 64 (1990) 2711.

[12] H. Nishimori, et al., J. Phys. Soc. Jpn. 73 (2004) 3247.

[13] A. Wachowiak, et al., Science 298 (2002) 577.

[14] S. S. Saxena, P. Monthoux, Nature 427 (2004) 799. 